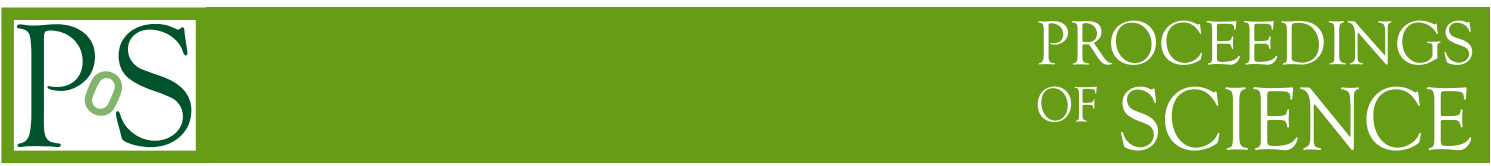

\title{
Solar neutrino results from Super-Kamiokande
}

\author{
Yuuki Nakano* for the Super-Kamiokande Collaboration \\ Particle Physics Group, Depertment of Physics, Kobe University \\ Rokkodai-cho, 1-1, Nada-ku, Kobe-city, Hyogo, 657-850, Japan. \\ E-mail: ynakano@phys.sci.kobe-u.ac.jp
}

\begin{abstract}
Super-Kamiokande (SK), a 50 kton water Cherenkov detector in Japan, observes ${ }^{8} \mathrm{~B}$ solar neutrinos with neutrino-electron elastic scattering. SK searches for distortions of the solar neutrino energy spectrum caused by the edge of the MSW resonance in the core of the Sun. It also searches for a day/night solar neutrino flux asymmetry induced by the matter in the Earth. The installation of new front-end electronics in 2008 marks the beginning of the 4th phase of SK (SK-IV). With the improvement of the water circulation system, calibration methods, reduction cuts, this phase achieved the lowest energy threshold thus far (3.5 MeV kinetic energy). SK observed solar neutrinos for about 19 years, that is 5,200 days. This long operation covers more than 1.5 solar activity cycles. An analysis about a possible correlation between solar neutrino flux and 11 year activity cycle will be presented. To test the matter effect induced by the Sun and the Earth, the energy spectrum and the day/night solar neutrino flux asymmetry from SK-I to SK-IV is analyzed. A global oscillation analysis using SK-I,II,III, and SK-IV data and combined with the results of other solar neutrino experiments as well as KamLAND reactor experiment has been carried out. The results of this global analysis will be presented as well.
\end{abstract}

38th International Conference on High Energy Physics

3-10 August 2016

Chicago, USA

${ }^{*}$ Speaker. 


\section{Introduction}

The solar neutrinos are produced by the nuclear fusion reaction, $4 p \rightarrow \alpha+2 e^{+}+2 v_{e}$, in the core of the Sun. Electron neutrinos produced in the Sun are so called $p p, p e p,{ }^{7} \mathrm{Be},{ }^{8} \mathrm{~B}$ and hep neutrinos, whose fluxes had been predicted by the standard solar model [1]. Their energy distributes from $\sim 0.1 \mathrm{MeV}$ to $\sim 20 \mathrm{MeV}$. The current interesting physics motivation of the solar neutrino observation with Super-Kamiokande (SK) [2] is to search for the Mikheyev-Smirnov-Wolfenstein (MSW) effect [3 4]. The MSW effect leads to a resonant conversion of the higher energy solar neutrinos within the Sun and results in an about 30\% level of the survival probability above a few $\mathrm{MeV}$ as shown in the left panel of Figure 1, which is so called "Up-turn". Since the energy spectrum reflects the survival probability of the electron neutrinos, SK searches for the "Spectrum up-turn" by measuring the recoil electron energy spectrum. In addition, due to the matter effect in the interior of the Earth, the electron flavor neutrinos are regenerated as shown in the right panel of Figure 1. It is expected that the neutrino flux in night is larger than that in day by about a few \% level depending on the neutrino oscillation parameters. In 2014, SK reported an indication of the terrestrial matter effects by about $2.7 \sigma[5]$.

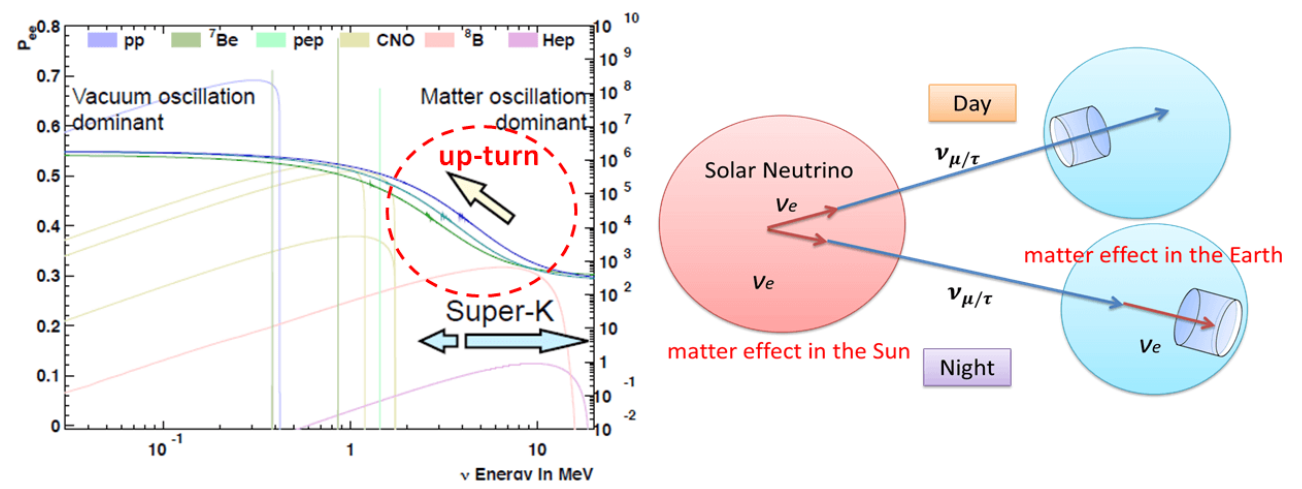

Figure 1: Left: Several prediction of the electron neutrino survival probability (left) with fluxes of each solar neutrino (right) as a function of the neutrino energy. Right: The visual explanation of the day/night flux asymmetry.

\section{Data analysis}

Recently, the Super-Kamiokande Collaboration released a paper about the solar neutrino analysis results [6]. In this proceedings, the updated results, expect for the day/night flux asymmetry measurement, are presented using data taken through the end of March 31st, 2016 (SK-IV 2365 days data set). The total livetime throughout the difference phases of SK [7, 9, is 5,200 days. By the way, the details of the analysis technique can be found in [6.

\subsection{Flux measurement}

The left panel of Figure 2 shows the distribution of cosine between the reconstructed direction of observed recoil electrons and the direction of the Sun. Using 5,200 days data, more than 84,000 
events are observed so far. Based on this data, the ${ }^{8} \mathrm{~B}$ solar neutrino flux is determined to be $(2.355 \pm 0.033) \times 10^{6}\left[/ \mathrm{cm}^{2} / \mathrm{sec}\right]$. The ratio between the SK result and the SNO NC current flux [10] becomes $0.4486 \pm 0.0062$. The solar activity cycle is the 11 years periodic change of sun spots releasing the magnetic flux at the surface of the Sun. They are strongly correlated with the solar activity cycle. SK observed solar neutrinos for about 19 years, this long term observation covers more than 1.5 solar activity cycles. The right panel of Figure 2 shows the SK yearly flux measured throughout the different phases of SK together with the corresponding Sun spot number (Source: WDC-SILSO, Royal Observatory of Belgium, Brussels [11). Using the present data, the $\chi^{2}$ is calculated with the total experimental error as $\chi^{2}=15.52 / 19$ d.o.f., which corresponds to a probability of $68.9 \%$. SK solar rate measurements are fully consistent with a constant solar neutrino flux emitted by the Sun.
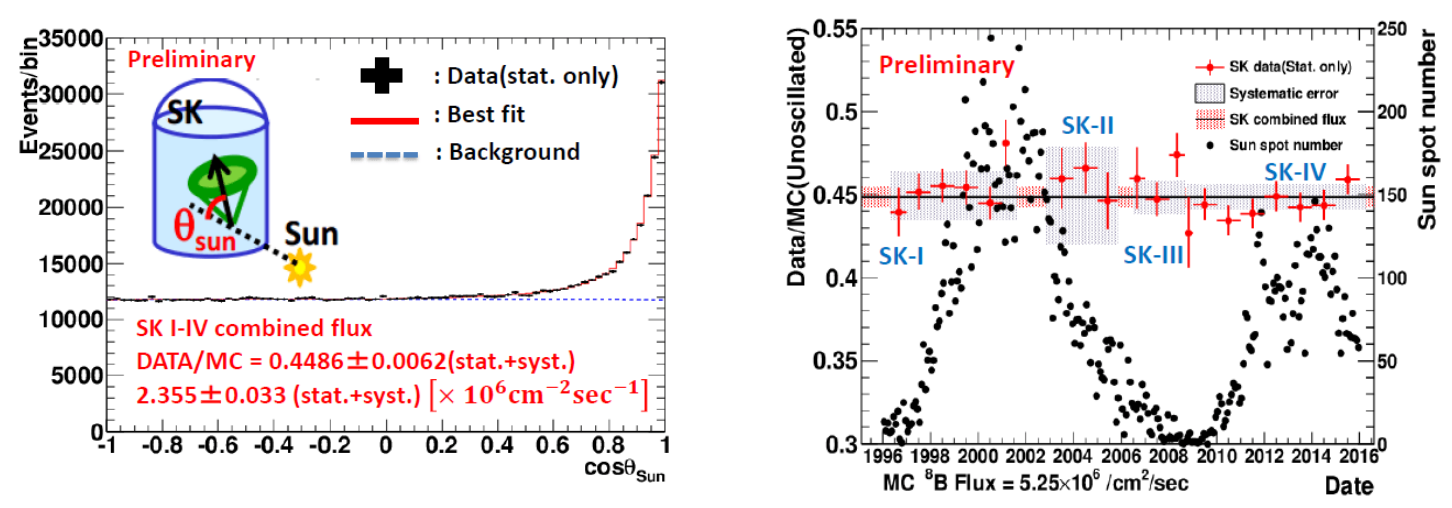

Figure 2: Left: The solar angle distribution combining for SK-I trough SK-IV. The horizontal axis shows the cosine of the solar angle $\cos \theta_{\text {Sun }}$ and the vertical axis shows the number of the observed events. The black points show the data, the red (blue) histogram shows the best-fit result (background-shape). Right: The result of the solar neutrino flux measurement from 1996 to 2015. The red points shows the yearly flux measured by SK (statistical error only), the gray bands shows the systematic uncertainties for each SK phase, the black-horizontal line shows the combined measured flux with the uncertainty drawn by the red band and the black points shows the sun spot number [11].

\subsection{Energy spectrum analysis}

The energy spectrum for the each SK phase are extracted using an extended maximum likelihood fit [6]. After that, they are compared with the expectations based on the MSW effect assuming two oscillation parameters obtained by a neutrino oscillation analysis discussed in subsection 2.3. The left panel of Figure 3 shows the combined energy spectrum with the predictions. Comparing $\chi^{2}$ between the data (black) and the predictions (green and blue), the MSW prediction drawn in blue is disfavored by $\sim 2 \sigma$ level. Neutrino energy spectrum is de-convoluted from the recoil electron energy spectrum. The right panel of Figure 3 shows the result of survival probability $\left(P_{e e}\left(E_{v}\right)\right)$ with the other experimental results ( $p p$ and $\mathrm{CNO}$ neutrino flux constraints from the all solar neutrino data [12,13, 14, 15], and the ${ }^{7} \mathrm{Be}$, pep and ${ }^{8} \mathrm{~B}$ flux measurements of Borexino [16, 17]). The red band shows the allowed region from SK plus SNO [10] combined data as a function of neutrino energy. This analysis result gives the world's strongest constraints on the shape of the survival probability in the transition region between vacuum oscillation and MSW resonance. 

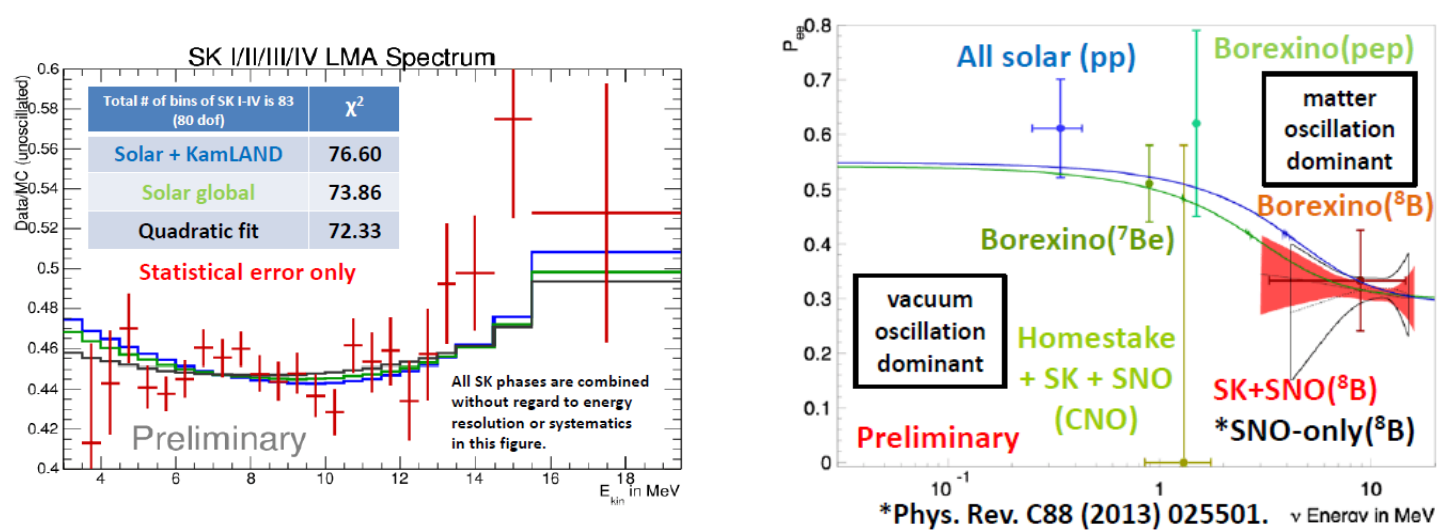

Figure 3: Left: The energy spectrum combining SK-I to SK-IV as a function of energy. The red points shows the ratio of the data to the expected flux using a non-oscillated ${ }^{8} \mathrm{~B}$ solar neutrino spectrum. The black histogram shows the best-fit result using quadratic function. The green (blue) histogram illustrates the MSW prediction based on the oscillation parameters from Solar global (Solar+KamLAND) $\sin ^{2} \theta_{12}=$ $0.311 \pm 0.014$ and $\Delta m_{21}^{2}=4.74_{-0.80}^{+1.40} \times 10^{-5} \mathrm{eV}^{-2}\left(\sin ^{2} \theta_{12}=0.308 \pm 0.012\right.$ and $\Delta m_{21}^{2}=7.49_{-0.18}^{+0.19} \times 10^{-5}$ $\mathrm{eV}^{-2}$ ). Right: The $1 \sigma$ band of the survival probability combining SK with SNO [10] as well as other measurements results 12, 13, 14, 15, 16, 17. The horizontal axis shows the neutrino energy and the vertical axis shows the electron neutrino survival probability.

\subsection{Neutrino oscillation analysis}

The oscillation analysis was conducted using SK, SNO [10], radiochemical solar neutrino experiments [12, 13, 14] and Borexino [15, 16, 17] as well as the anti-neutrino measurement by KamLAND [18, 19]. SK uniquely selects the Large Mixing Angle MSW region by more than $3 \sigma$ and SK significantly contributes to the measurement of the solar angle $\theta_{12}$. When combining with SNO data, it gives strongest constraints on $\sin ^{2} \theta_{12}$ as shown in the left panel of Figure 4 (green dash-line). On the other hand, The SK spectrum and day/night data favors a lower $m_{21}^{2}$ value than KamLAND's by more than $2 \sigma$. This parameter is mostly determined by the solar neutrino oscillation fit and further precise measurements are required in future. Removing the $\theta_{13}$ constraint, oscillation angle $\theta_{13}$ can be extracted using the solar neutrino experiments as well as the KamLAND as shown in the right panel of Figure 4. The measured value is $\sin ^{2} \theta_{13}=0.029_{-0.015}^{+0.014}$, which is $2 \sigma$ level away from zero. The obtained result is consistent with the precise reactor measurement [20, 21,22].

\section{Summary}

Super Kamiokande has precisely measured the ${ }^{8} \mathrm{~B}$ solar neutrino flux, its time variation and recoil electron spectrum. No significant correlation between the observed solar neutrino flux and the sun spot number is found. In addition, the energy spectrum analysis slightly disfavors the MSW prediction by $\sim 2 \sigma$ level. In the solar neutrino oscillation analysis combined with the KamLAND result, oscillation parameters are determined as $\Delta m_{21}^{2}=7.49_{-0.18}^{+0.19} \times 10^{-5} \mathrm{eV}^{2}, \sin ^{2} \theta_{12}=0.308 \pm$ 0.012 . When the constrain of $\theta_{13}$ is removed, solar neutrino experiments and KamLAND measure $\sin ^{2} \theta_{13}=0.029_{-0.015}^{+0.014}$, the value is good agreement with reactor anti-neutrino measurements. 

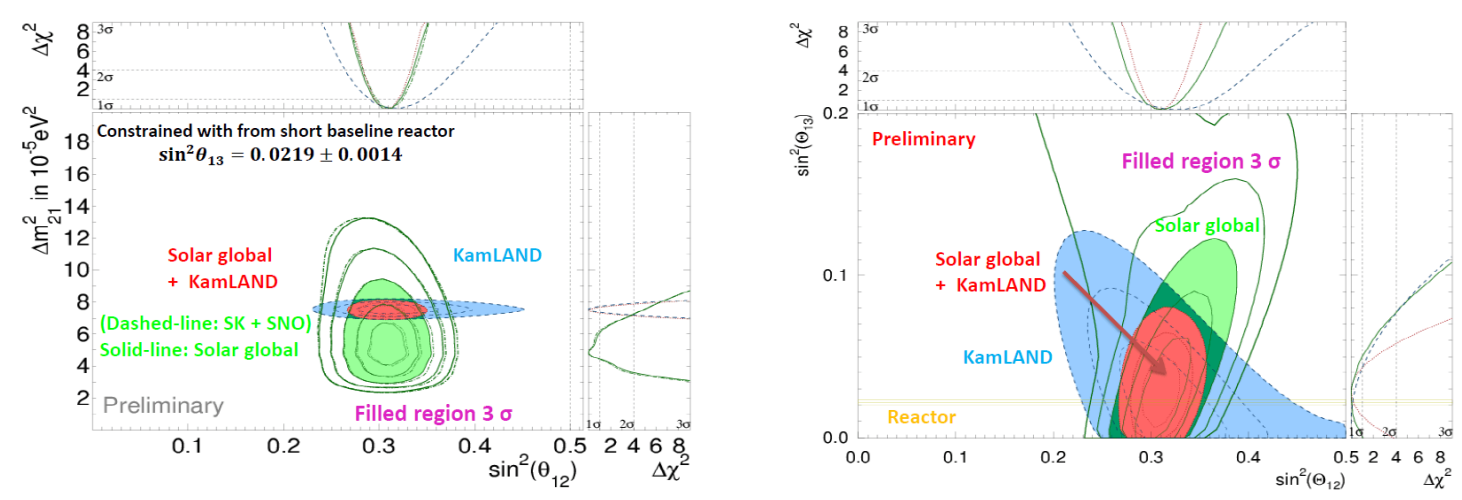

Figure 4: Left: Allowed contours for $\Delta m_{21}^{2}$ vs. $\sin ^{2} \theta_{12}$ from the combination of SK and SNO [10] (greendashed) at 1, 2, 3, 4 and $5 \sigma$ confidence levels. In addition, solar neutrino data (green-solid) and KamLAND data (blue) [18 19] are also shown. The all combined allowed region is drawn in red. Th filled regions gives the $3 \sigma$ confidence levels. Right: Allowed contours of $\sin ^{2} \theta_{13}$ vs. $\sin ^{2} \theta_{12}$, where colors are the same as the left panel. The yellow band shows the results from the reactor anti-neutrino measurements [20,21, 22].

\section{References}

[1] John N. Bahcall and Roger K. Ulrich, Rev. mod, Phys. 60, 297 (1988).

[2] Y. Fukuda et al., Nucl. Instrum. Meth. A 501, 418 (2003).

[3] S. P. Mikheyev and A. Y. Smirnov, Sov. Jour. Nucl. Phys. 42, 913 (1985).

[4] L. Wolfenstein, Phys. Rev. D 17, 2369 (1978).

[5] A. Renshaw et al., Phys. Rev. Lett. 112, 091805 (2014).

[6] K. Abe et al., Phys. Rev. D 94, 052010 (2016).

[7] J. Hosaka et al., Phys. Rev. D 73, 112001 (2006).

[8] J. P. Cravens et al., Phys. Rev. D 78, 032002 (2008).

[9] K. Abe et al., Phys. Rev. D 83, 052010 (2011).

[10] B. Aharmin et al., Phys. Rev. C 88, 025501 (2013).

[11] WDC-SILSO, Royal Observatory of Belgium, Brussels. http://www.sidc.be/silso/datafiles

[12] R. Davis, Jr., D. S. Harmer, and K. C. Hoffman et al., Phys. Rev. Lett. 20, 1205 (1968).

[13] J. N. Adburashitov et al., Phys. Rev. C 80, 015807 (2009).

[14] M. Altmann et al., Phys. Lett. B 616, 174 (2005).

[15] G. Bellini et al., Phys. Rev. Lett. 107, 141302 (2011).

[16] Bellini et al., Phys. Rev. D 82, 033006 (2010).

[17] Bellini et al., Phys. Rev. Lett. 707, 051302 (2012).

[18] S. Abe et al., Phys. Rev. Lett 100, 221803 (2008).

[19] A. Gando et al., Phys. Rev. D 88, 033001 (2013).

[20] F. P. An et al., Chin. Phys. C 37, 011001 (2013).

[21] J. K. Ahn et al., Phys. Rev. Lett. 108, 191802 (2012).

[22] Y. Abe et al., Phys. Rev. D 86, 052008 (2012). 